\title{
Evaluación de factores de riesgo asociados a dehiscencia anastomótica en cirugía colorrectal. Análisis multivariado de $\mathbf{7 4 8}$ pacientes
}

\author{
Nelson Muñoz P. ${ }^{1}$, Marcelo Rodríguez G. ${ }^{1,2}$, Alberto Pérez-Castilla ${ }^{1,2}$, \\ Nicolás Campaña W. ${ }^{3}$ y Gonzalo Campaña V. ${ }^{1,2}$
}

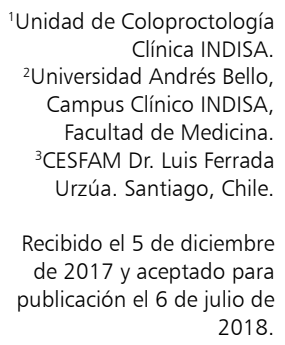

Correspondencia a:

Dr. Gonzalo Campaña V. gonzalo.campana@indisa.cl
Evaluation of the risk factors associated with anastomotic dehiscence in colorectal surgery. Multivariate analysis of 748 pacients

Introduction: Anastomotic leakage (AL) is a severe complication in colorectal surgery, its incidence ranges from 2 to $19 \%$. In international literature, we found numerous studies on the identification of risk factors (RF), while in the national there are only two series that analyze this complication. Aim: Perform a descriptive characterization of institutional results and establish the AL rate, its associated risk factors and mortality. Materials and Method: Non-concurrent series of cases, whose sample is consecutive patients operated for colorectal pathology with primary anastomosis with or without a derivative ostoma between 2004 and 2016. Univariate and multivariable logistic regression model was performed. Results: There were 748 patients, $50.5 \%$ women, mean age was 56.2 . The most frequent surgical indications were colorectal cancer in $381(50.9 \%)$ patients and diverticular disease in $163(21.8 \%)$. The AL was $5.6 \%(42 / 748)$ and the mortality was $2 \%(15 / 748)$, being $1 \%$ for the electives $(7 / 681)$. In the univariate analysis, we found that the RF that had statistical significance were albumin $(p<0.001)$, anastomosis height $(p<0.001)$, transfusion $(p<0.001)$, location (right colon $>$ left $)(p=0.011)$, while that in the multivariate analysis were albumin $(\mathrm{p}=0.002)$ with an OR $3.64($ IC 95\% 1.58-8.35) and transfusion $(\mathrm{p}=0.015)$ with an OR 7.15 (IC 95\% 1.46-34.91). Conclusion: Our series is the largest reported in Chile, with similar results to international and national studies. We establish that hypoalbuminemia and the presence of intraoperative transfusions are associated with a high rate of AL.

Key words: anastomotic leak; colorectal surgery; risk factors.

\section{Resumen}

Introducción: La dehiscencia anastomótica (DA) es una complicación severa en cirugía colorrectal con una incidencia que oscila entre 2 y $19 \%$. La literatura internacional muestra numerosos estudios sobre la identificación de factores de riesgo (FR), mientras que en la nacional existen solo dos series que analizan esta complicación. Objetivo: Realizar una caracterización descriptiva de resultados institucionales y establecer la tasa de DA, sus factores de riesgo asociados y la mortalidad. Materiales y Método: Serie de casos no concurrente, cuya muestra son pacientes consecutivos intervenidos de patología colorrectal con anastomosis primaria con o sin ostoma derivativo entre los años 2004 y 2016. Se realiza modelo de regresión logística univariable y multivariable. Resultados: Se obtuvieron 748 pacientes, 50,5\% mujeres, media de edad fue 56,2. Las indicaciones quirúrgicas más frecuentes fueron cáncer colorrectal en 381 $(50,9 \%)$ pacientes y enfermedad diverticular en $163(21,8 \%)$. La DA fue de $5,6 \%(42 / 748)$ y la mortalidad fue de $2 \%(15 / 748)$, siendo de $1 \%$ para los electivos (7/681). En el análisis univariado encontramos que los FR que tuvieron significancia estadística fueron la albúmina $(\mathrm{p}<0,001)$, altura anastomosis $(\mathrm{p}<0,001)$, transfusión $(\mathrm{p}<0,001)$, localización (colon derecho $>$ izquierdo) $(\mathrm{p}=0,011)$, mientras que en el análisis multivariado fueron la albúmina $(\mathrm{p}=0,002)$ con un OR 3,64 (IC 95\% 1,58-8,35) y transfusión $(\mathrm{p}=0,015)$ con un OR 7,15 (IC 95\% 1,46-34,91). Conclusión: Nuestra serie es la más grande reportada en Chile, con resultados similares a estudios internacionales y nacionales. Establecemos que la hipoalbuminemia y la presencia de transfusiones intraoperatorias se asocian a alta tasa de DA.

Palabras clave: dehiscencia anastomótica; cirugía colorrectal; factores de riesgo. 


\section{Introducción}

La dehiscencia anastomótica (DA) es una complicación severa en cirugía colorrectal. Su incidencia varía ampliamente en las publicaciones entre un $1,8-19 \%{ }^{1-5}$ siendo mayor en cirugía de recto. Este amplio rango se debe en parte a la variabilidad de conceptos y definición de $\mathrm{DA}^{6,7}$, a los criterios de inclusión considerados en las publicaciones y a que existen diferencias en tasa de DA según tipo de resección y anastomosis. Se debe considerar también la existencia de múltiples factores de riesgos (FR) interrelacionados que afectan directamente en el resultado ${ }^{8,9}$. Dentro de los FR clásicos asociados a DA está la cirugía de recto (altura anastomosis), neoadyuvancia, sexo masculino, transfusiones y comorbilidades como la obesidad, hábito tabáquico y alcohólico, desnutrición y uso de corticoides.

Con la aparición de suturas mecánicas y su masificación en los últimos 30 años, ha generado un cambio en la cirugía colorrectal, permitiendo realizar anastomosis más bajas con conservación de esfinter ${ }^{10}$, con resultados similares a las anastomosis manuales en anastomosis colorrectales ${ }^{11}$.

La DA puede llevar a sepsis, reoperaciones y en algunos casos conlleva mortalidad asociada. En pacientes oncológicos produce retardo en el inicio de la quimioterapia, disminución de la sobrevida y aumento en la recurrencia ${ }^{12,13}$

En la literatura internacional, existen numerosos estudios sobre el tema e identificación de $\mathrm{FR}^{1,4,5,14-16}$, mientras que en la nacional hay sólo dos series publicadas en 2006 y $2016^{17,18}$.

Creemos que es fundamental conocer y transparentar nuestros resultados, debido a que la DA es un indicador de calidad importante en cirugía colorrectal.

El objetivo principal es realizar una caracterización descriptiva de resultados institucionales por primera vez tabulados. Los objetivos secundarios son establecer la tasa de DA, sus factores de riesgo asociados y la mortalidad a 60 días.

\section{Pacientes y Método}

Corresponde a una serie de casos no concurrente, cuya muestra son pacientes consecutivos intervenidos de patología colorrectal en Clínica INDISA. Los criterios de inclusión y exclusión se presentan en Tabla 1, incluyéndose pacientes inmunosuprimidos y con cirugías colónicas previas; se excluyó el traumatismo colónico por accidente de tránsito, arma de fuego o blanca. No hubo lesiones iatrogénicas en esta serie.

Nuestra variable dependiente es la presencia de DA y las variables independientes son los factores de riesgo para ella. Éstas las dividimos en relacionadas al paciente y relacionadas con la cirugía. En las primeras incluimos edad, sexo, IMC, ASA, comorbilidades (hipertensión arterial, diabetes mellitus tipo 2, enfermedad renal, hepática, pulmonar o cardíaca, uso de anticoagulantes), hábito tabáquico y alcohol, laboratorio (albúmina y hemoglobina), uso de corticoides, laparotomías previas, preparación intestinal y neoadyuvancia. En las variables relacionadas con la cirugía consideramos la indicación quirúrgica, tipo de cirugía realizada, de urgencia o electiva, tipo de cirujano, abordaje, características anastomosis, presencia de ostoma derivativo, tiempo quirúrgico, pérdida de sangre, transfusión de glóbulos rojos, cirugía concomitante, reoperación, infección de herida operatoria y mortalidad a 60 días.

Definimos DA como la acontecida en pacientes, que en contexto de un posoperatorio anormal tengan: 1) salida de contenido intestinal por drenaje, herida operatoria o fístula a órgano vecino; 2) pacientes reintervenidos en quienes se confirmó DA en el intraoperatorio por presencia de filtración, peritonitis localizada (colección) o generalizada o 3) tomografía computada que evidenció salida de contraste, colección o burbuja perianastomótica ${ }^{18,19}$.

Se realizó una revisión de registros clínicos electrónicos y ficha física para construir una base de datos en Excel $^{\circledR}$ con información codificada y anonimizada. Ésta fue realizada por dos investigadores y cuando existía alguna duda se resolvía con un cirujano senior.

Tabla 1. Criterios de inclusión y exclusión

\begin{tabular}{|l|l|}
\hline Criterios de inclusión & Criterios de exclusión \\
\hline Cirugía colorrectal & Menores de 15 años \\
\hline \multicolumn{1}{|c|}{ Hemicolectomía derecha } & Trauma \\
\hline Transversectomía & Datos incompletos \\
\hline Hemicolectomía izquierda & \\
\hline Sigmoidectomía & \\
\hline RAB o RAUB & \\
\hline Colectomía total & \\
\hline Reconstitución tránsito & \\
Anastomosis primaria & \\
Con o sin ostoma derivativo & \\
\hline Período enero de 2004 a diciembre de 2016 & \\
\hline
\end{tabular}




\section{ARTÍCULO ORIGINAL}

La estadística utilizada fue descriptiva y analítica. Las variables categóricas se expresaron en números y porcentajes con cálculo de $\chi^{2}$ y las continuas en medias \pm DS, con aplicación de prueba de Mann-Whitney para variables sin distribución normal.

Se realizaron análisis de regresión logística univariaday multivariada para corregir posibles factores de confusión, utilizando un modelo por pasos con la inclusión de variables significativas considerando un alfa $<0,05$ en el análisis univariada previo y con posterior cálculo de Odds Ratio e intervalos de confianza al 95\%. El análisis de los datos se realizó con software STATA 13.0.

Este estudio cuenta con la aprobación del Comité

Tabla 2. Perfil biodemográfico y características cirugía

\begin{tabular}{|c|c|c|}
\hline \multicolumn{2}{|l|}{ Característica } & $n=748$ \\
\hline \multicolumn{2}{|c|}{ Edad (años) media \pm DS } & $56,2 \pm 16,1$ \\
\hline \multirow{2}{*}{ Sexo n $(\%)$} & Masculino & $370(49,5 \%)$ \\
\hline & Femenino & $378(50,5 \%)$ \\
\hline \multirow[t]{4}{*}{ ASA } & I & $239(32 \%)$ \\
\hline & II & $402(53,7 \%)$ \\
\hline & III & $96(12,8 \%)$ \\
\hline & IV & $11(1,5 \%)$ \\
\hline \multirow[t]{4}{*}{ IMC } & $<20$ & $32(4,2 \%)$ \\
\hline & $20-25$ & $358(48 \%)$ \\
\hline & $26-30$ & $279(37,3 \%)$ \\
\hline & $>30$ & $79(10,5 \%)$ \\
\hline \multirow[t]{2}{*}{ Tipo cirugía } & Electiva & $681(91,1 \%)$ \\
\hline & Urgencia & $67 \quad(8,9 \%)$ \\
\hline \multirow[t]{2}{*}{ Tipo cirujano } & General & $519(69,3 \%)$ \\
\hline & Coloproctólogo & $229(30,7 \%)$ \\
\hline \multirow[t]{3}{*}{ Abordaje } & Abierto & $298(39,8 \%)$ \\
\hline & Laparoscópico & $439(58,7 \%)$ \\
\hline & Robótico & $11(1,4 \%)$ \\
\hline \multirow[t]{2}{*}{ Tipo anastomosis } & Manual & $155(20,7 \%)$ \\
\hline & Mecánica & $593(79,3 \%)$ \\
\hline \multirow[t]{8}{*}{ Tipo cirugía } & Sigmoidectomía & $323(43,2 \%)$ \\
\hline & Hemicolectomía derecha & $216(28,8 \%)$ \\
\hline & RAB + RAUB + RAUBIE* & $91(12 \%)$ \\
\hline & Reconstitución operación Hartmann & $65(8,6 \%)$ \\
\hline & Colectomía total & $27 \quad(3,6 \%)$ \\
\hline & Transversectomía & $15(2 \%)$ \\
\hline & Panproctocolectomía + reservorio & $6(0,8 \%)$ \\
\hline & TaTME** & $5(0,6 \%)$ \\
\hline
\end{tabular}

*RAB: Resección Anterior Baja, RAUB: Resección Anterior Ultrabaja, RAUBIE: Resección Anterior Ultrabaja Interesfintérica. **TaTME: Escisión mesorrectal total transanal. de Bioética de la Universidad Andrés Bello, respetando los principios éticos de la Declaración de Helsinki ${ }^{20}$ y Taipéi ${ }^{21}$.

\section{Resultados}

Se obtuvieron un total de 861 pacientes, excluyendo 113 por no cumplir criterios de inclusión; de éstos, a 96 pacientes se les realizó ostomía terminal sin anastomosis primaria (operación de Hartmann o similar), 14 resecciones en cuña de ciego y 3 casos en que no se obtuvo ficha clínica, quedando finalmente 748 pacientes para el análisis.

La media de edad fue $56,2 \pm 16,1$, de las cuales $50,5 \%$ eran mujeres.

Las indicaciones quirúrgicas fueron cáncer de colon en $302(40,4 \%)$ pacientes, cáncer de recto en $79(10,5 \%)$, carcinoides apendiculares en 14 $(1,8 \%)$, otros cáncer con compromiso colónico en $15(2 \%)$, enfermedad diverticular en $163(21,8 \%)$, reconstitución operación de Hartmann en $65(8,7 \%)$, endometriosis en $28(3,7 \%)$, enfermedad inflamatoria intestinal en $11(1,5 \%)$, inercia colónica en 11 $(1,5 \%)$, vólvulo cecal y sigmoides en $21(2,8 \%)$, poliposis adenomatosa familiar en $2(0,2 \%)$ y otros en 37 (4,9\%) (apendicitis aguda complicada, invaginación intestinal, perforaciones intestinales, megacolon, tuberculosis colónica, tumores desmoides). La cirugía fue electiva en $91 \%$, realizada vía laparoscópica en 58,7\%, con una conversión de 15,8\% y con anastomosis mecánica en 79,3\%. La cirugía más realizada fue la sigmoidectomía con un $43,2 \%$, seguida de la hemicolectomía derecha en un $28,8 \%$ y cirugía rectal en un $12 \%$. El perfil biodemográfico de los pacientes, así como las características de la cirugía, se exponen en Tabla 2.

Se realizaron 486 cirugías izquierdas, de ellas la anastomosis fue intraperitoneal en el $88,5 \%$ (430/486). Las ostomías de protección estuvieron presentes en el 9,9\% (48/486) del total de las cirugías izquierdas, siendo un 2\% (9/430) para las anastomosis intraperitoneales y un $69 \%(39 / 56)$ para las extraperitoneales $(\mathrm{p}<0,001)$. No hubo diferencias significativas al comparar la presencia de FA en quienes tuvieron ostoma protector $4,1 \%(2 / 46)$ con quienes no lo tuvieron 4,9\% $(24 / 483)(p=0,852)$.

La DA global fue de 5,6\% (42/748), ocurriendo entre el día 1 y 35 posoperatorio, con una moda de pesquisa al $4^{\circ}$ día posoperatorio. Los resultados de DA según la ubicación de la anastomosis se muestran en Tabla 3 y su terapia en Figura 1. Hubo 3 casos en que se realizó tratamiento médico conservador con reposo intestinal, nutrición paren- 
Tabla 3. Dehiscencia anastomótica

\begin{tabular}{|llcr|}
\hline Dehiscencia anastomótica global & $5,6 \%$ & $(42 / 748)$ \\
Ileocólica & & $6,9 \%$ & $(15 / 216)$ \\
Colorrectal & Global & $4,5 \%$ & $(22 / 486)$ \\
& Intraperitoneal & $3,0 \%$ & $(13 / 430)$ \\
& Extraperitoneal & $16 \%$ & $(9 / 56)$ \\
Ileorrectal & & $12 \%$ & $(3 / 25)$ \\
Coloanal & & $13,3 \%$ & $(2 / 15)$ \\
Ileoanal & & $0 \%$ & $(0 / 6)$ \\
\hline
\end{tabular}

teral y antibiótico endovenoso. De los pacientes que tuvieron DA, el 92\% requirió una reoperación como tratamiento. Hubo 8 casos en que se realizó una ileostomía terminal posterior a una anastomosis ileocólica dehiscente. Hubo 5 casos en que se realizó una nueva anastomosis, todas fueron anastomosis ileocólicas, filtrando nuevamente 3 de ellas en que luego se realizó ileostomía terminal.

$\mathrm{Al}$ evaluar la técnica quirúrgica y DA según tipos específicos de configuraciones anastomóticas (Tabla 4), no encontramos diferencias estadísticamente significativas en ninguna de las variables analizadas. Cabe mencionar que las anastomosis ileocólicas fueron en su mayoría (65\%) laterolaterales (LL) anisoperistálticas con sutura mecánica (técnica de Barcelona) y el resto fueron isoperistálticas manuales, ya sea LL, término-laterales (TL) o términoterminales (TT).

La mortalidad global fue de $2 \%$ (15/748), siendo un $1 \%$ para los electivos $(7 / 681)$ y un $11,9 \%(8 / 67)$ para urgencia $(\mathrm{p}<0,001)$. De los fallecidos, un

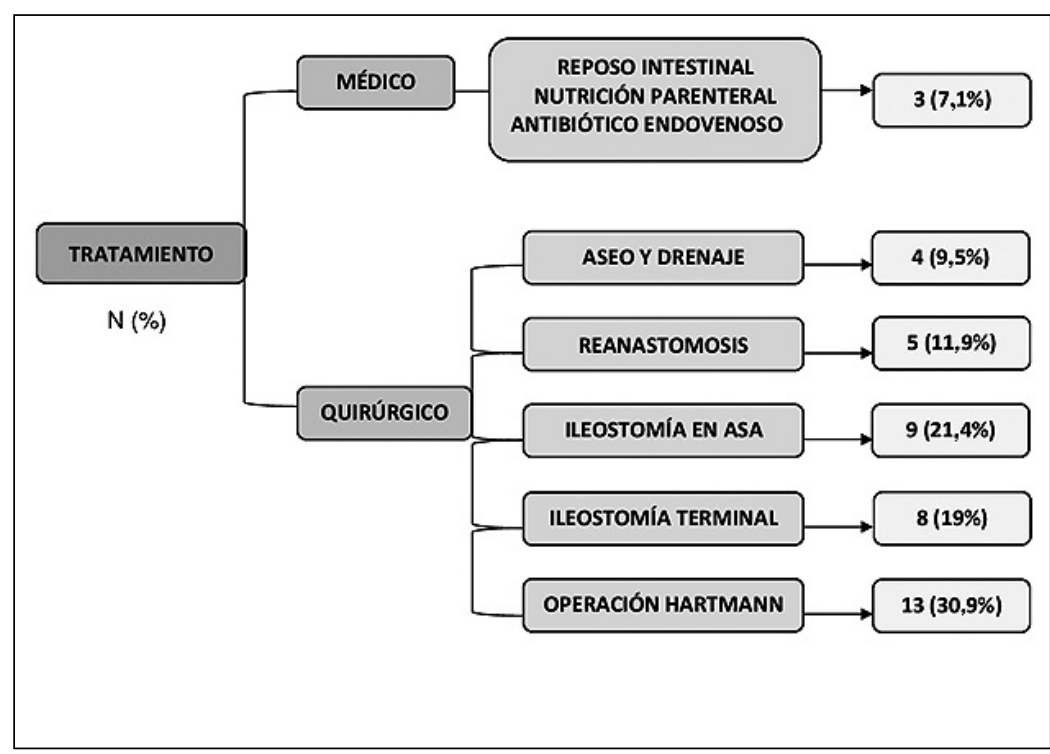

Figura 1. Tratamiento de pacientes con filtración anastomótica.

$80 \%(12 / 15)$ fue por causa directa, es decir, sepsis secundaria a DA. Los otros fallecidos (3/15) fueron por causas médicas ( 2 neumonías asociada a atención en salud y 1 coagulopatía). La mortalidad en el grupo de pacientes que presentaron DA fue de $28,5 \%(12 / 42)$ y aquellos sin DA de un $0,4 \%$ $(3 / 706)(\mathrm{p}<0,001)$.

Se reoperó el 8,9\% (67/748), siendo la DA el motivo de reintervención en un 58\% (39/67). Los otros motivos de reoperación fueron 6 hemoperitoneos, 5 cirugías revisionales sin hallazgos significativos, 4 obstrucciones intestinales, 3 hematomas de la pared, 3 colecciones sin DA, 2 perforaciones

Tabla 4. Técnica quirúrgica y DA según tipos específicos de anastomosis

\begin{tabular}{|c|c|c|c|c|}
\hline & & & iscencia anastom & \\
\hline & & Ileocólica & Co & ctal \\
\hline & & & Intraperitoneal & Extraperitoneal \\
\hline Sutura & Manual & $4,1 \%(3 / 74)$ & $3 \% \quad(2 / 67)$ & $0 \%(0 / 1)$ \\
\hline & Mecánica & $8,4 \%(12 / 142)$ & $2,7 \%(10 / 363)$ & $16 \%(9 / 55)$ \\
\hline Lateralidad & LL & $8,1 \%(14 / 173)$ & $\mathrm{NA}^{*}$ & NA* \\
\hline & $\mathrm{TL}$ & $2,7 \%(1 / 37)$ & $1,8 \%(1 / 55)$ & $0 \%(0 / 6)$ \\
\hline & TT & $0 \% \quad(0 / 6)$ & $2,9 \%(11 / 375)$ & $18 \%(9 / 49)$ \\
\hline Peristaltismo & Anisoperistáltica & $7,1 \%(11 / 154)$ & $\mathrm{NA}^{*}$ & $\mathrm{NA}^{*}$ \\
\hline & Isoperistáltica & $6,4 \%(4 / 62)$ & $\mathrm{NA}^{*}$ & $\mathrm{NA}^{*}$ \\
\hline Diagnóstico & Maligno & $7,7 \%(12 / 156)$ & $2,3 \%(4 / 176)$ & $16 \%(7 / 43)$ \\
\hline & Benigno & $5 \% \quad(3 / 60)$ & $3,2 \%(8 / 254)$ & $15 \%(2 / 13)$ \\
\hline
\end{tabular}

NA*: No aplica análisis. No hubo diferencias estadísticamente significativas en ninguna de las variables expuestas en Tabla. 
Tabla 5. Asociación de variables continuas relacionadas al paciente y cirugía con dehiscencia anastomótica (prueba de Mann-Whitney)

\begin{tabular}{|c|c|c|c|}
\hline Variable & Con DA $(n=42)$ & $\operatorname{Sin} \mathrm{DA}(n=706)$ & Valor $\mathbf{p}$ \\
\hline $\begin{array}{l}\text { Relacionada al paciente } \\
\text { Edad (años) } \\
\mathrm{IMC}\left(\mathrm{Kg} / \mathrm{mt}^{2}\right) \\
\mathrm{Hb}(\mathrm{g} / \mathrm{dL}) \\
\text { Creatinina }(\mathrm{mg} / \mathrm{dL}) \\
\text { Albúmina }(\mathrm{g} / \mathrm{dL})\end{array}$ & $\begin{aligned} 59,88 & \pm 16,52 \\
25,62 & \pm 3,62 \\
12,27 & \pm 2,01 \\
0,825 & \pm 0,24 \\
3,23 & \pm 0,89\end{aligned}$ & $\begin{array}{r}56,03 \pm 16,20 \\
25,75 \pm 4,33 \\
12,96 \pm 2,36 \\
0,884 \pm 0,65 \\
3,86 \pm 0,64\end{array}$ & $\begin{aligned} & 0,1120 \\
& 0,9149 \\
& 0,025 \\
& 0,7104 \\
< & 0,001\end{aligned}$ \\
\hline
\end{tabular}

Tabla 6. Asociación de variables categóricas relacionadas al paciente con dehiscencia anastomótica $\left(\chi^{2}\right)$

\begin{tabular}{|c|c|c|c|c|}
\hline Variable & & Con DA & Sin DA & Valor $\mathbf{p}$ \\
\hline Sexo & $\begin{array}{l}\text { Masculino } \\
\text { Femenino }\end{array}$ & $\begin{array}{l}27 \\
15\end{array}$ & $\begin{array}{l}345 \\
361\end{array}$ & 0,052 \\
\hline Tabaco & $\begin{array}{l}\text { Sí } \\
\text { No }\end{array}$ & $\begin{array}{l}19 \\
21\end{array}$ & $\begin{array}{l}237 \\
428\end{array}$ & 0,130 \\
\hline Alcohol & $\begin{array}{l}\text { Sí } \\
\text { No }\end{array}$ & $\begin{array}{l}11 \\
29\end{array}$ & $\begin{array}{l}176 \\
487\end{array}$ & 0,895 \\
\hline Corticoides & $\begin{array}{l}\text { Sí } \\
\text { No }\end{array}$ & $\begin{array}{r}0 \\
40\end{array}$ & $\begin{array}{r}11 \\
653\end{array}$ & 0,412 \\
\hline Neoadyuvancia* & $\begin{array}{l}\text { Sí } \\
\text { No }\end{array}$ & $\begin{array}{l}7 \\
2\end{array}$ & $\begin{array}{l}31 \\
33\end{array}$ & 0,099 \\
\hline HTA** $^{* *}$ & $\begin{array}{l}\text { Sí } \\
\text { No }\end{array}$ & $\begin{array}{l}18 \\
22\end{array}$ & $\begin{array}{l}252 \\
410\end{array}$ & 0,381 \\
\hline $\mathrm{DM} 2 * *$ & $\begin{array}{l}\text { Sí } \\
\text { No }\end{array}$ & $\begin{array}{r}6 \\
34\end{array}$ & $\begin{array}{r}84 \\
580\end{array}$ & 0,666 \\
\hline $\mathrm{ERC}^{* *}$ & $\begin{array}{l}\text { Sí } \\
\text { No }\end{array}$ & $\begin{array}{r}0 \\
40\end{array}$ & $\begin{array}{r}19 \\
643\end{array}$ & 0,277 \\
\hline Enf. hepática & $\begin{array}{l}\text { Sí } \\
\text { No }\end{array}$ & $\begin{array}{r}1 \\
39\end{array}$ & $\begin{array}{r}6 \\
657\end{array}$ & 0,324 \\
\hline Enf. cardíaca & $\begin{array}{l}\text { Sí } \\
\text { No }\end{array}$ & $\begin{array}{r}5 \\
35\end{array}$ & $\begin{array}{r}63 \\
601\end{array}$ & 0,510 \\
\hline Enf. pulmonar & $\begin{array}{l}\text { Sí } \\
\text { No }\end{array}$ & $\begin{array}{r}7 \\
33\end{array}$ & $\begin{array}{r}68 \\
595\end{array}$ & 0,150 \\
\hline TACO & $\begin{array}{l}\text { Sí } \\
\text { No }\end{array}$ & $\begin{array}{r}2 \\
38\end{array}$ & $\begin{array}{r}18 \\
645\end{array}$ & 0,399 \\
\hline Laparotomías previas & $\begin{array}{l}\text { Sí } \\
\text { No }\end{array}$ & $\begin{array}{l}16 \\
24\end{array}$ & $\begin{array}{l}271 \\
386\end{array}$ & 0,876 \\
\hline Preparación intestinal & $\begin{array}{l}\text { Sí } \\
\text { No }\end{array}$ & $\begin{array}{r}32 \\
5\end{array}$ & $\begin{array}{l}520 \\
136\end{array}$ & 0,289 \\
\hline ASA & $\begin{array}{l}\text { I } \\
\text { II } \\
\text { III } \\
\text { IV }\end{array}$ & $\begin{array}{r}13 \\
22 \\
4 \\
1\end{array}$ & $\begin{array}{r}212 \\
393 \\
57 \\
5\end{array}$ & 0,393 \\
\hline
\end{tabular}

*Neoadyuvancia, sólo para cáncer de recto medio e inferior. **HTA: hipertensión arterial, DM2: diabetes mellitus tipo 2, ERC: enfermedad renal crónica, TACO: tratamiento anticoagulante. de intestino delgado, y retención gástrica, hernia interna, vólvulo gástrico, dehiscencia cúpula vaginal y evisceración con una en cada caso. En el subgrupo de pacientes reintervenidos que presentaron DA, esta segunda operación se pudo llevar a cabo vía laparoscópica en el 34,8\% (8/23) de los pacientes en que la cirugía previa fue realizada por este abordaje.

La infección de sitio operatorio (ISO) estuvo presente en el 2,1\% (16/748), siendo un $1,5 \%$ $(11 / 706)$ en pacientes sin DA y un $11,9 \%(5 / 42)$ para pacientes con DA $(\mathrm{p}<0,001)$. Esta complicación se presentó solo en un caso de cirugía laparoscópica.

La media de estadía hospitalaria en los pacientes con DA fue de 18,3 $\pm 4,5$ días, mientras que en aquellos sin DA fue de 6,9 $\pm 4,5$ días $(p<0,001)$.

$\mathrm{Si}$ clasificamos a los pacientes en dos grupos, aquellos con DA y sin DA, las asociaciones de factores de riesgo relacionadas al paciente y cirugía se revelan en Tablas 5, 6 y 7; destacando con significancia estadística, en las variables relacionadas al paciente, la hemoglobina preoperatoria $(p=0,025)$ y la albúmina preoperatoria $(\mathrm{p}<0,001)$. En las variables relacionadas a la cirugía destacan, la altura de la anastomosis $(\mathrm{p}<0,001)$ y la transfusión de glóbulos rojos intraoperatoria $(\mathrm{p}<0,001)$.

Al realizar un modelo de regresión logística con un análisis univariado (Tabla 8), evaluando las variables que se asociaron a DA, encontramos que los FR que tuvieron significancia estadística fueron la albúmina $(\mathrm{p}<0,001)$, altura anastomosis $(\mathrm{p}<0,001)$, transfusión $(\mathrm{p}<0,001)$ y localización (colon derecho $>$ izquierdo) $(\mathrm{p}=0,011)$. Con el mismo modelo de regresión logística en el análisis multivariado establecemos que aquellos con significancia estadística fueron la albúmina $(\mathrm{p}=0,002)$ con un OR 3,64 (IC 95\% 1,58-8,35) y transfusión $(\mathrm{p}=0,015)$ con un OR 7,15 (IC 95\% 1,46-34,91). 
ARTíCULO ORIGINAL

Existen otras variables asociadas a DA, que sin tener significancia estadística en el análisis de regresión logística son dignos de considerar, como son el sexo $7,8 \%$ vs $4,1 \%(\mathrm{p}=0,052)$ de DA para masculino y femenino respectivamente, terapia neoadyuvante $22,5 \%$ vs $6 \%$ sin terapia $(\mathrm{p}=0,099)$ $\mathrm{y}$ una hemoglobina preoperatoria baja $(\mathrm{p}=0,025)$.

La periodicidad y temporalidad del estudio en relación a la DA y mortalidad se refleja en Figura 2, donde queda de manifiesto una clara tendencia a la disminución de ambas complicaciones en los últimos años, incluso con ausencia de mortalidad en los últimos 4 años y con DA cercana al 5\% los últimos 3 años.

\section{Discusión}

Son bien conocidas las consecuencias potencialmente devastadoras de la DA luego de una colectomía en términos morbimortalidad, esto asociado a un aumento en la estadía hospitalaria y costos en la atención en salud ${ }^{22}$.
Tabla 7. Asociación de variables categóricas relacionadas a la cirugía con dehiscencia anastomótica $\left(\chi^{2}\right)$

\begin{tabular}{|llccc|}
\hline Variable & & Con DA & Sin DA & Valor p \\
Transfusión & Sí & 22 & 104 & \\
Cirugía urgencia & So & 18 & 556 & $<0,001$ \\
& Só & 3 & 64 & \\
Cirugía & Sí & 39 & 633 & 0,655 \\
concomitante & No & 12 & 200 & \\
Abordaje & Abierto & 30 & 506 & 0,972 \\
& Laparoscópico & 18 & 279 & \\
Tipo anastomosis & Manual & 8 & 428 & 0,572 \\
& Mecánica & 34 & 146 & \\
Ostoma protector & Sí & 2 & 560 & 0,799 \\
& No & 24 & 459 & 0,805 \\
Altura recto & Superior & 1 & 39 & \\
& Medio & 7 & 33 & $<0,001$ \\
& Inferior & 3 & 14 & \\
Tipo cirujano & General & 27 & 483 & 0,576 \\
& Coloproctólogo & 15 & 223 & 0,56 \\
\hline
\end{tabular}

Tabla 8. Evaluación de factores de riesgo independiente de dehiscencia anastomótica mediante modelo de regresión logística con análisis univariado y multivariado

\begin{tabular}{|lcccccc|}
\hline Variable & \multicolumn{3}{c}{ Regresión logística univariada } & \multicolumn{3}{c|}{ Regresión logística multivariada } \\
& $\mathbf{p}$ & $\mathbf{O R}$ & $\mathbf{I C ~ 9 5 \%}$ & $\mathbf{p}$ & $\mathbf{O R}$ & $\mathbf{I C}$ 95\% \\
\hline Hemoglobina $(\mathrm{g} / \mathrm{dL})$ & 0,087 & 0,88 & $0,76-1,01$ & 0,078 & 1,30 & $0,97-1,74$ \\
\hline Albúmina $(\mathrm{g} / \mathrm{dL})$ & $<0,001$ & 2,96 & $1,76-5,00$ & 0,002 & 3,64 & $1,58-8,35$ \\
\hline Altura anastomosis & $<0,001$ & 0,23 & $0,10-0,49$ & 0,052 & 0,10 & $0,01-1,01$ \\
Transfusión & $<0,001$ & 6,53 & $3,38-12,6$ & 0,015 & 7,15 & $1,46-34,9$ \\
\hline
\end{tabular}

OR: Odds Ratio.

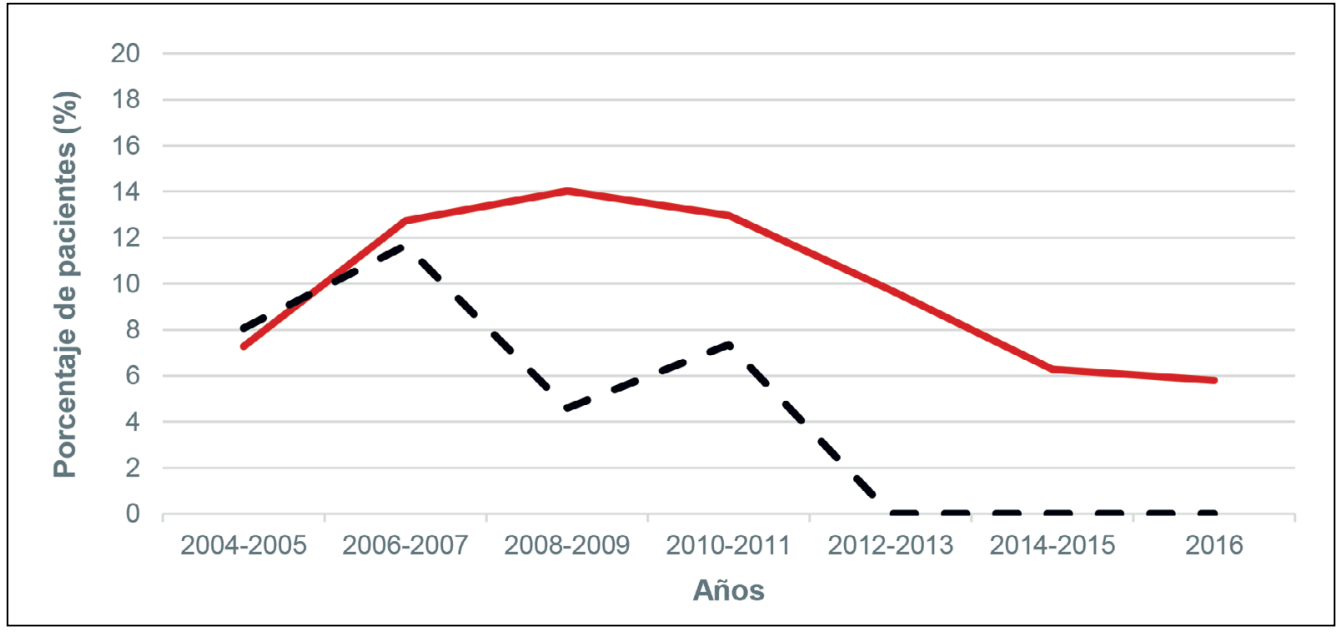

Rev. cir. 2019;71(2):136-144
Figura 2. Gráfico de porcentaje de dehiscencia anastomótica y mortalidad según período de tiempo (DA: línea roja continua, mortalidad: línea negra punteada). 
Nuestra serie da cuenta de la experiencia con mayor número de pacientes publicada en Chile, con resultados similares a los reportados en la literatura internacional ${ }^{1,14-16,22,23}$ y nacional ${ }^{17,18}$. En términos de tasa global de DA las publicaciones nacionales reportan un $3,8 \%$ y un $6,7 \%{ }^{17,18}$, similar al $5,6 \%$ reportado por nosotros.

Llama la atención que en nuestra serie la DA ileocólica sea mayor a la colorrectal, 6,9\% vs 4,5\%, respectivamente. Sin embargo, nuestro resultado es prácticamente similar al 7,9\% reportado por una serie nacional recientemente publicada ${ }^{18}$, no así con el $1 \%$ de filtración reportada por otro reporte nacional previo ${ }^{17}$. No obstante, las dos series europeas más grandes que evalúan la anastomosis ileocólica obtienen un $8,4 \%{ }^{24}$ y $8,1 \%{ }^{25}$ de DA, mientras que reportes norteamericanos revelan un $3,7 \%{ }^{5}$. Incluso esta mayor DA tiene repercusión en una mayor mortalidad al compararlo con el colon izquierdo ${ }^{14}$, dejando atrás el concepto en que clásicamente se ha menospreciado la anastomosis ileocólica.

La mortalidad global de la serie fue de un $2 \%$ (15/748), dando cuenta de un 28\% (12/42) para pacientes con DA y un $0,4 \%(3 / 706)$ para el grupo sin DA $(p<0,001)$, lo que es esperable y concordante con algunas series que dan cuenta de hasta el $30 \%$ de mortalidad en aquellos pacientes con filtración ${ }^{14}$.

Queremos destacar que no existieron diferencias estadísticamente significativas en el riesgo de presentar DA al evaluar la presencia de comorbilidades y ASA (Tabla 6), asociados históricamente como factores de riesgo de complicación quirúrgica. Otros estudios también han mostrado resultados inconsistentes relacionados con estas variables ${ }^{16,26}$.

Al igual que otras series ${ }^{27}$, el impacto clínico de la DA, queda demostrado por el mayor tiempo de hospitalización, la mayor tasa de reoperaciones y mortalidad.

A partir de nuestros resultados podemos establecer que los FR locales asociados a DA en el análisis univariado, relacionados con el paciente, son la hipoalbuminemia y hemoglobina baja preoperatoria, mientras que los FR relacionados a la cirugía son la altura de la anastomosis, (recto inferior y medio mayor que recto superior) la ubicación de la anastomosis (ileocólica mayor a colorrectal) y la presencia de transfusiones intraoperatorias.

El análisis multivariado muestra que la hipoalbuminemia y la presencia de transfusiones intraoperatorias se asocian con una alta tasa de DA. Ambas reportadas en otros estudios ${ }^{12,21,24,25}$. Diversas series han demostrado que la hipoalbuminemia (nivel de albúmina menor a $3,5 \mathrm{~g} / \mathrm{dL}$ ) es un factor de riesgo independiente de $\mathrm{DA}^{29,30}$, siendo en nuestra serie de $3,23 \pm 0,89$ con un OR de 3,64 , esto significa que aquellos pacientes con hipoalbuminemia tienen 3,64 veces más chances de presentar DA que aquellos con albúmina normal y que aquellos con transfusiones tienen 7,15 veces más chances de presentar DA que aquellos sin transfusiones.

Al igual que otros autores ${ }^{16,22,28}$, no demostramos que la cirugía realizada de urgencia tuviera impacto en la DA $(p=0,655)$. En nuestro estudio la cirugía de urgencia fue realizada en el 8,9\% (67/748) del total de pacientes, representando el 7\% (3/42) de las DA. Sin embargo, sí encontramos que tiene un impacto en la mortalidad, según lo ya descrito.

Entre las limitaciones mencionar, por una parte, que la muestra es unicéntrica y, por otro lado, al diseño retrospectivo del estudio. Sin embargo, también tiene fortalezas como el tamaño muestral y el buen registro clínico, ya que en todos los casos se obtuvo los datos principales (DA y mortalidad), y que en el $94 \%$ estaban registradas la totalidad de las variables estudiadas. Por otro lado, consideramos que la heterogeneidad de la muestra constituye una fortaleza, ya que al incluir pacientes electivos y de urgencia, así como también oncológicos y benignos, produce un acercamiento a la realidad. Además, al considerar la periodicidad y temporalidad del estudio vemos una mejoría en los resultados de DA y mortalidad, lo que podríamos interpretar como una consolidación y maduración en el desarrollo local de la cirugía colorrectal, respecto a la experiencia inicial.

\section{Conclusión}

Nuestra serie es la más grande reportada en Chile, con resultados comparables a estudios internacionales y nacionales. Establecemos que la hipoalbuminemia y la presencia de transfusiones intraoperatorias se asocian a alta tasa de DA. La relevancia clínica de la DA queda evidenciada en el mayor tiempo de hospitalización, la mayor tasa de reoperaciones y mortalidad, al compararla con pacientes sin DA.

Es necesario crear un estudio prospectivo, aleatorizado e idealmente multicéntrico con un número aún mayor de pacientes, donde se analicen las distintas estrategias de confección de anastomosis ilecólicas, para poder dar una respuesta más sólida a nuestros resultados respecto a la mayor tasa de filtración de éstas en comparación a las colorrectales intraperitoneales. 


\section{Responsabilidades éticas}

Protección de personas y animales. Los autores declaran que para esta investigación no se han realizado experimentos en seres humanos ni en animales.
Confidencialidad de los datos. Los autores declaran que en este artículo no aparecen datos de pacientes.

Conflictos de interés: no hay.

\section{Referencias}

1. Frasson M, Flor-Llorente B, Ramos JL, Granero-Castro P, Hervás D, Álvarez MA, et al. Risk Factors for Anastomotic Leak After Colon Resection for Cancer. Ann Surg. 2015;262:321-30.

2. Asteria C, Gagliardi G, Pucciarelli S, Romano G, Infantino A, La Torre F, et al. Anastomotic leaks after anterior resection for mid and low rectal cancer: survey of the Italian Society of Colorectal Surgery. Tech Coloproctol. 2008;12:103-10.

3. Matthiessen $P$, Hallböök $\mathrm{O}$, Rutegård J, Simert G, Sjödahl R. Defunctioning Stoma Reduces Symptomatic Anastomotic Leakage After Low Anterior Resection of the Rectum for Cancer. Ann Surg. 2007;246:207-14.

4. McDermott FD, Heeney A, Kelly ME, Steele RJ, Carlson GL, Winter DC. Systematic review of preoperative, intraoperative and postoperative risk factors for colorectal anastomotic leaks. Br J Surg. 2015;102:462-79.

5. Parthasarathy $M$, Greensmith M, Bowers D, Groot-Wassink T. Risk factors for anastomotic leakage after colorectal resection: a retrospective analysis of 17 518 patients. Color Dis. 2017;19:288-98.

6. Bruce J, Krukowski ZH, Al-Khairy G, Russell EM, Park KGM. Systematic review of the definition and measurement of anastomotic leak after gastrointestinal surgery. Br J Surg. 2001;88:1157-68.

7. Rahbari N, Weitz J, Hohenberger W, Heald R, Moran B, Ulrich A, et al. Definition and grading of anastomotic leakage following anterior resection of the rectum: A proposal by the International Study Group of Rectal Cancer. Surgery 2010;147:339-51.

8. Daams F, Luyer M, Lange JF. Colorectal anastomotic leakage: Aspects of prevention, detection and treatment. World J Gastroenterol. 2013;19:2293-7.

9. Suding P, Jensen E, Abramson M, Itani
K, Wilson S. Definitive Risk Factors for Anastomotic Leaks in Elective Open Colorectal Resection. Arch Surg. 2008;143:907-12.

10. Bannura G, Melo C, Contreras J, Barrera A, Villaman JJ, Lee KS. Suturas mecánicas en cirugía colorrectal: análisis de 225 pacientes consecutivos. Rev Chi Cir. 2002;54:350-7.

11. Neutzling CB, Lustosa SA, Proenca IM, da Silva EM, Matos D. Stapled versus handsewn methods for colorectal anastomosis surgery. Cochrane Database Syst Rev. 2012;1-51.

12. Goto $S$, Hasegawa $S$, Hida K, Uozumi R, Kanemitsu Y, Watanabe T, et al. Multicenter analysis of impact of anastomotic leakage on long-term oncologic outcomes after curative resection of colon cancer. Surgery. 2017;162:317-24.

13. Law WL, Choi HK, Lee YM, Ho J, Seto CL. Anastomotic Leakage is Associated with Poor Long-Term Outcome in Patients After Curative Colorectal Resection for Malignancy. J Gastrointest Surg. 2007;11:8-15.

14. Bakker IS, Grossmann I, Henneman $\mathrm{D}$, Havenga $\mathrm{K}$, Wiggers T. Risk factors for anastomotic leakage and leakrelated mortality after colonic cancer surgery in a nationwide audit. Br J Surg. 2014;101:424-32.

15. Alves A, Panis Y, Trancart D, Regimbeau J, Pocard M, Valleur P. Factors associated with clinically significant anastomotic leakage after large bowel resection: Multivariate analysis of 707 patients. World J Surg. 2002;26:499-502.

16. Buchs N, Gervaz P, Secic M, Bucher P, Mugnier-Konrad B, Morel P. Incidence, consequences, and risk factors for anastomotic dehiscence after colorectal surgery: A prospective monocentric study. Int J Colorectal Dis. 2008;23:265-70.

17. Bannura G, Cumsille M, Barrera A, Contreras. J, Melo C, Soto D, et al.
Factores asociados a la dehiscencia clínica de una anastomosis intestinal grapada: análisis multivariado de 610 pacientes consecutivos. Rev Chil Cir. 2006;58:3416.

18. López-Köstner F, Cerda C, Wainstein C, Kronberg U, Larach A, Larach J, et al. Impacto de las filtraciones anastomóticas en cirugía colorrectal. Rev Chi Cir. 2016;68:417-21.

19. Peel A, Taylor E. Proposed definitions for the audit of postoperative infection: a discussion paper The report of a workshop convened by the following participants: Surgical Infection Study Group with the. Ann R Coll Surg Engl. 1991;73:385-8.

20. World Medical Association. World Medical Association Declaration of Helsinki. Ethical Principles for Medical Research Involving Human Subjets. Jama. 2013;310:2191-4.

21. Asociación Médica Mundial. Declaración de Taipei sobre Consideraciones Éticas de las bases de datos de salud y Biobancos. Asociación médica mundial [Internet]. Ferney-Voltaire, Francia [citado el 4 de diciembre de 2017]. Disponible desde: https://www.wma.net/es/policies-post/ declaracion-de-la-amm-sobre-lasconsideraciones-eticas-de-las-bases-dedatos-de-salud-y-los-biobancos/.

22. Leichtle SW, Mouawad NJ, Welch KB, Lampman RM, Cleary RK. Risk factors for anastomotic leakage after colectomy. Dis Colon Rectum. 2012;55:569-75.

23. Trencheva K, Morrissey KP, Wells M, Mancuso CA, Lee SW, Sonoda T, et al. Identifying Important Predictors for Anastomotic Leak After Colon and Rectal Resection. Ann Surg. 2013;257:108-13.

24. Frasson M, Granero-Castro P, Ramos Rodríguez JL, Flor-Lorente B, Braithwaite M, Martí Martínez E, et al. Risk factors for anastomotic leak and postoperative morbidity and mortality after elective right colectomy for cancer: results from a prospective, multicentric study 


\section{ARTÍCULO ORIGINAL}

of 1102 patients. Int J Colorectal Dis. 2016;31:105-14.

25. European Society of Coloproctology collaborating group. The relationship between method of anastomosis and anastomotic failure after right hemicolectomy and ileo-caecal resection: an international snapshot audit. Color Dis. 2017;12:3218-21.

26. Konishi T, Watanabe T, Kishimoto J, Nagawa $\mathrm{H}$. Risk factors for anastomotic leakage after surgery for colorectal cancer:
Results of prospective surveillance. J Am Coll Surg. 2006;202:439-44.

27. Choi HK, Law WL, Ho JWC. Leakage after resection and intraperitoneal anastomosis for colorectal malignancy: Analysis of risk factors. Dis Colon Rectum. 2006;49:1719-25.

28. Biondo S, Parés D, Kreisler E, Ragué JM, Fraccalvieri D, Ruiz AG, et al. Anastomotic dehiscence after resection and primary anastomosis in left-sided colonic emergencies. Dis Colon Rectum
2005;48:2272-80.

29. Telem D, Chin E, Nguyen S, Divino C. Risk Factors for Anastomotic Leak Following Colorectal Surgery. Arch Surg. 2010;145:371-6.

30. Hennessey D, Burke J, Ni-Dhonochu T, Shields C, Winter D, Mealy K. Preoperative Hypoalbuminemia is an Independent Risk Factor for the Development of Surgical Site Infection Following Gastrointestinal Surgery. Ann Surg. 2010;252:325-9. 